\title{
Measurement of the B0 ---> Psi (2S) Lambda0 Branching Fraction on BaBar at the Stanford Linear Accelerator Center
}

by Alexander Raymond Olivas, Jr.

Ph.D. Thesis 
Olivas, $\mathrm{Jr}_{r}$, Alexander Raymond (Ph. D, Physics)

Measurement of the $B^{0} \rightarrow \psi(2 S) K^{0}$ Branching Fraction on BaBar at the Stanford Linear Accelerator Center.

Thesis directed by Professor Uriel Nanenberg

The decays of $B^{0}$ mesons to hadronic final states remains a rich area of physics on BaBar. Not only do the $c \pi \mathrm{K}$ final states $\left(\mathrm{e}, \mathrm{g}, B^{0} \rightarrow \psi^{\prime}(2 S) K^{0}\right)$ allow for the measurement of CP Violation, but the branching fractions provide a sensitive test of the theoretical methods used to account for low energy non-pertarbative QCD effects.

We present the measurement of the branching fraction for the decay $B^{0} \rightarrow \psi(2 S) K_{\text {a }}$. The data set consists of $88.8 \pm 1.0 \times 10^{6} B \bar{B}$ pairs collected on the $e^{+} e^{-} \rightarrow \Upsilon(4 S)$ resonance on BaBar/PEP-Il at the Stanford Linear Acoelerator Center (SLAC). This analysis features a modification of present cuts, with respect to those publisthed so far on BaBar, on the $K_{\mathrm{s}} \rightarrow \pi^{+} \pi^{-}$ and $\psi(2 S) \rightarrow J / \psi \pi^{+} \pi^{-}$which aim at reducing the background while keeping the signal intact. Various data selection criteria are studied for the lepton modes $\left(e^{+} e^{-}\right.$and $\left.\mu^{+} \mu^{-}\right)$of the $J / \psi$ and $\psi(2 S)$ to improve signal purity as well as study the stability of the resultant branching fractions. 


\section{Contents}

\section{Chapter}

1 Introduction 1

2 The Standard Model $\quad 2$

2.1 A Brief History of $20 t$ Century Physics . . . . . . . . . . 2

2.2 Fundamental Particles . . . . . . . . . . . . . . . . . 4

2.2 .1 The fermions ......................... 4

2.2 .2 The Gauge Bosons $\ldots \ldots \ldots \ldots \ldots$

2.3 The Cabibbo-Kobayashi-Maskawa Matrix . . . . . . . . . . . . 6

3 Physics of $e^{+} e^{-}$collisions at the $\mathrm{T}(4 S)$ Resonance $\quad 9$

4 Theoretical Estimate of the branching fraction 12

5 PEP-II and The BaBar Detector 15

5.1 The PEP-II Collider $\ldots \ldots \ldots \ldots \ldots \ldots \ldots \ldots$

5.2 The BaBar Magnet and Flux Return . . . . . . . . . . . . . . . . . . . 19

5.3 The Silicon Vertex Tracker , , , , . . . . . . , . . . . . . . . . 21

5.3 .1 Principles of Silicon Strip Sensors . . . . . . . . . . . . . . 21

5.3 .2 Layout of the BaBar Silicon Vertex Tracker . . . . . . . . . . 21

5.3 .3 Performance of the BaBar Silicon Vertex Tracket . . . . . . . . . 23

5.4 The Drift Chamber . . . . . . . . . . . . . . . . . . . . . . 23

3.4 .1 Principle of Operation . . . . . . . . . . . . . . . . 23

5.42 The BaBar Drift Charuber . . . . . . . . . . . . . . . . 26

5.4 .3 Performance of the BaBar Drift Chamber . . . . . . . . . 26

5.5 Performance of combined BaBar tracking system (SVT and DCH) . . . . 29 
5.6 The Detection of Internally Rellected Cherenkov light . . . . . . . . . . . 29

5.6 .1 Principle of Operation . . . . . . . . . . . . . . . 31

5.6.2 The BaBar DIRC . . . . . . . . . . . . . . . . . . 32

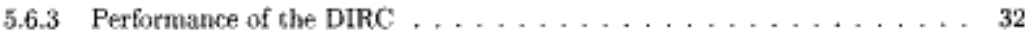

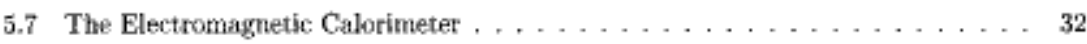

5.7 .1 Principle of Operation $\ldots \ldots \ldots \ldots \ldots \ldots \ldots . \ldots \ldots$

5.7 .2 The BaBar Electromagnetic Calorimeter . . . . . . . . . . . . . . . 35

5.7 .3 Performance of the BaBar EMC . . . . . . . . . . . . . . 35

5.8 The Instruruented Flux Return . . . . . . . . . . . . . . . . . . . . . . . 39

5.8 .1 Principle of Operation . . . . . . . . . . . . . 39

5.8 .2 The BaBar Instramented Flux Return . . . . . . . . . . . . . . . 40

5.8.3 Performance of the Instrumented Flux Return . . . . . . . . . . . . 40

6 Analysis Introduction

6.1 Analysis Method . . . . . . . . . . . . . . . . . . . . . 44

6.2 Description of the data set . . . . . . . . . . . . . . . . . 45

$\begin{array}{lll}7 & \text { Background Event Topology } & 47\end{array}$

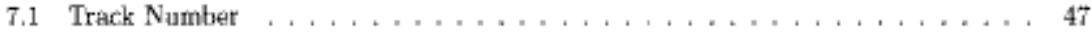

7.2 Total Energy . . . . . . . . . . . . . . . . . . . . . . . . 49

7.3 Fox-Wolfram Moments . . . . . . . . , . . . . . . . . . . . . . . . 49

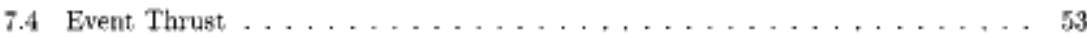

7.5 Initjal Data $\mathrm{Skim} \ldots \ldots \ldots \ldots \ldots \ldots \ldots \ldots \ldots$

$8 \quad K_{S} \rightarrow \pi^{+} \pi^{-}$- Selection and Reconstruction Study

8.1 Monte Carlo Track Finding Study . . . . . . . . . . . . . . . . . . 58

8.2 Decay length and momentum collinearity $\ldots \ldots \ldots \ldots \ldots, \ldots, \ldots, \ldots$

8.3 Combined $K_{s}$ Cuts . . . . . . . . . . . . . . . . 60

8.4 Comparison to Generic Monte Carlo . . . . . . . . . . . . . . . . . . . 62

9 Tracking Efficiency and Selection of $J / \psi \rightarrow l^{+} l^{-}$and $t(2 S) \rightarrow l^{+} l^{-}$

9.1 Monte Carlo Track Finding Efficiency . . . . . . . . . . . . . . . . . . 66

9.2 Particle Identification $\ldots \ldots \ldots \ldots, \ldots, \ldots, \ldots, \ldots, \ldots, \ldots, \ldots, \ldots$ 
$10 \psi(2 S) \rightarrow J / \psi \pi^{4} \pi^{-}$- Tracking Efficiency and Selection Analysis 71

10.1 Tracking Efficiency $\ldots \ldots \ldots \ldots \ldots \ldots \ldots$

10.2 Mass difference between the $\psi(2 S)$ and $d / \psi$ candidates $\ldots \ldots \ldots \ldots$. . . . 71

10.3 Remowal of $\psi(2 S) \rightarrow J / \psi \pi^{+} \pi^{-}$candidates which share pions daughters with

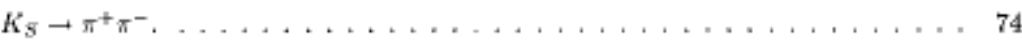

11 Systematic Study of the Branching Fraction $B^{0} \rightarrow \psi(2 S) K^{0} \quad 78$

11.1 The $t(2 S) \rightarrow l^{+} t^{-}$Final Stales $\ldots \ldots \ldots \ldots \ldots \ldots . \ldots \ldots$

11.2 The $\psi(2 S) \rightarrow J / \psi^{+} \pi^{-}$Final States . . . . . . . . . . . . 78

$12 B^{0}$ Analysis and Branching Fraction Results 82

12.1 Reconstruction Efficiency in Signal Monte Carlo . . . . . . . . . . . 82

12.2 The Standard BaBar $B^{0} \rightarrow \psi(2 S) K_{S}$ Selection $\ldots \ldots \ldots \ldots \ldots \ldots$

12.2 .1 Inproving the Standard Selection $\ldots \ldots \ldots \ldots . \ldots \ldots$

12.3 Branching Fraction Results $\ldots \ldots \ldots \ldots \ldots \ldots \ldots$. . . . . . . . . . 84

12.3 .1 Systematic Errors $\ldots . . . \ldots \ldots 4 . \ldots \ldots$

12.3 .2 Branching Fraction . . . . . . . . . . . . . . . . . 87

13 Conclusion

Bibliography 90

\section{Appendix}

A Defirition of the Jpsitoll Skin

A.1 Background Multi-Hadron Filter $\ldots \ldots \ldots, \ldots, \ldots \ldots, \ldots, \ldots, \ldots, 92$

A.2 B-Counting Mutj-Hadron filter $\ldots \ldots \ldots, \ldots, \ldots, \ldots 2$

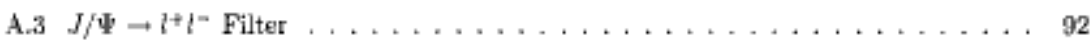

B $B^{0}$ Meson Kinematics

B.1 Derivation of Energy substituted $B^{0}$ Mass $\ldots \ldots \ldots \ldots \ldots \ldots$. . . . . . .

C Particle Identification (PID) Selectors 97

C.1 Description of the variables used in electron identification $\ldots \ldots \ldots \ldots .97$

C.2 Elextron Likelithood Selector . . . . . . . . . . . . . . . . . . . . 98 
C.3 Muon Cut Based Selector . . . . . . . . . . . . . . . . 98

C.3.1 Description of the variables used for muon identification . . . . . . . . 98

D Description of Candidate Lists 100

D.1 Definition of GoodTracksLoose . . . . . . . . . . . . . . . . . . 100

D.2 Definition of ChargedTracks . . . . . . . . . . . . . . . . . 100

E Theta distribution of $\psi$ lepton daughters not reconstructed 101

F Particle ID Selection efficiencies for $J / \psi \rightarrow t^{+} t^{-}$and $\phi(2 S) \rightarrow 1^{+} t^{-}$

F.1 Monte Carlo Corrections . . . . . . . . . . . . . . . . . . . . . 103

G Selection of $B^{0}$ Candidates for $m_{\psi(2 S)}-m_{J / v}$ Study $\quad 109$

H The BaBar Standard $B^{0}$ Meson Selection 110

I $B$ neson $\Delta E$ and $m_{E S}$ plots for signal Monte Carlo, generic $B \bar{B}$ plus continuum Monte

$\begin{array}{ll}\text { Carlo, and data. } & 112\end{array}$

I.1 $m_{E S}$ Plots . . . . . . . . . . . . . . . . . . . . . . 112

1.1.1 Plots used in the calculation of the branching fraction . . . . . . . 112

1.1.2 Extreme cases considered in the estimation of the systematic error . . . 114

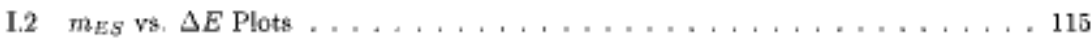

J Plots of Fractional Deviations of branching fractions for Various Selection Criteria $\quad 116$ 